\title{
Quasiparticle random phase approximation predictions of the gamma-ray strength functions using the Gogny force
}

\author{
Stéphane Hilaire ${ }^{1, a}$, Stéphane Goriely ${ }^{2}$, Sophie Péru ${ }^{1}$, François Lechaftois ${ }^{1}$, Isabelle Deloncle ${ }^{1,3}$, and Marco Martini ${ }^{4}$ \\ ${ }^{1}$ CEA, DAM, DIF, 91297 Arpajon, France \\ 2 Institut d'Astronomie et d'Astrophysique, Université Libre de Bruxelles, CP-226, 1050 Brussels, Belgium \\ ${ }^{3}$ CSNSM, CNRS and Université Paris-Sud, 91405 Orsay Campus, France \\ ${ }^{4}$ ESNT, CEA, IRFU, Service de Physique Nucléaire, Université de Paris-Saclay, 91191 Gif-sur-Yvette, France
}

\begin{abstract}
Dipole excitations of nuclei are crucial since they play an important role in nuclear reaction modeling in connection with the photoabsorption and the radiative capture processes. We present here results for the gamma-ray strength function obtained in large-scale axially-symmetric deformed quasiparticle (qp) random phase approximations approach using the finite-range Gogny force, with a particular emphasis on the E1 mode. The convergence with respect to the number of harmonic oscillator shells adopted and the cut-off introduced in the 2-quasiparticle excitation energy space is analyzed. The microscopic nature of our selfconsistent Hartree-Fock-Bogoliubov plus QRPA (HFB+QRPA) calculation has unfortunately to be broken, some phenomenological corrections being needed to take into account effects beyond the standard 2-qp QRPA excitations and the coupling between the single-particle and low-lying collective phonon degrees of freedom. The corresponding phenomenological parameters are adjusted on experimental photoabsorption data. In such a procedure, a rather satisfactory description of experimental data is obtained. To study the sensitivity of these phenomenological corrections on the extrapolation, both at low energies and towards exotic neutronrich nuclei, three different prescriptions are considered. They are shown to lead to rather similar predictions of the E1 strength at low energies as well as for exotic neutron-rich nuclei. The Gogny-HFB+QRPA strength is finally applied to the calculation of radiative neutron capture cross sections and the predictions compared with those obtained with more traditional Lorentzian-type approaches.
\end{abstract}

\section{Introduction}

Photonuclear and neutron capture data, describing interactions of photons and neutrons with atomic nuclei, are of importance for a variety of applications covering radiation shielding, radiation transport analyses, human body radiotherapy, activation analyses, safeguards, inspection technologies, nuclear waste transmutation, fission and fusion reactor technologies as well as astrophysical processes of nucleosynthesis. Within this framework, the knowledge of photon strength functions (PSF) is mandatory to describe both the average response of the nucleus to an electromagnetic probe or to estimate the gamma decay probability in competition with all other open channels. In most of the case, Lorentzian based phenomenological expressions are used with more or less refinements [1] introduced to improve the fit of available experimental data. However the number of nuclei for which PSF data are available remains rather weak compared to the number of nuclei involved in nucleosynthesis applications, and even when data are available, the energy range covered by the measurement is often too restricted to ensure a confident extrapolation of arbitrary phenomenological expressions. For this reason, theoretical approaches based on sound physical bases are of great importance to fill the gaps by either providing PSF far from experimentally accessible regions or by

a e-mail: stephane.hilaire@cea.fr offering a mean to test the reliability or the deviations from phenomenological expressions in unmeasured energy ranges. Indeed, provided satisfactory reproduction of available experimental data, the more microscopic the underlying theory, the greater the confidence in the extrapolations towards the experimentally unreachable regions. Microscopic approaches are rarely used for practical applications for several reasons. First, the computing time is often prohibitive for large-scale calculations. Second, the fine tuning required to reproduce accurately a large experimental data set is far from easy, in addition to being also time consuming. The only exception is represented by Refs. [2,3] in which a complete set of PSF based on mean field plus quasiparticle random-phase approximation (QRPA) calculations has been obtained and tabulated to be used in a nuclear reaction code. However, in Refs. [2,3], zero range Skyrme forces were considered and phenomenological corrections have been applied to properly describe the splitting of the giant dipole resonance (GDR) in deformed nuclei as well as the damping of the collective motion. The present study aims to go beyond the former approximation exploiting the axially symmetric-deformed QRPA approach based on Hartree-Fock-Bogoliubov (HFB) calculations using the finite-range Gogny interaction in a fully consistent way. This approach has proven its ability to reproduce qualitatively [4] and more recently, quantitatively thanks to few empirical corrections [5] a large set of experimental 
data. A large scale systematic calculation of the PSF is thus presently under consideration.

\section{Sensitivity analysis}

In order to perform systematic calculation within an accessible computing time, a compromise based on convergence analysis has been found to be necessary [5]. It consists in optimising the choice of the number of harmonic oscillator shells (HO), $N_{s h}$, over which the single-particle wave functions are expanded simultaneously with the maximum energy of the 2-quasiparticle states (called cutoff). The sensitivity to the Gogny interaction has also been considered, using both the D1S [6] and D1M [7] set of parameters. Both interactions have been found to provide rather similar results, but only $\mathrm{D} 1 \mathrm{M}$ has been used for large scale calculations considering its better accuracy with respect to experimental masses [7]. Typical results of the sensitivity analysis are illustrated in Fig. 1. As can be observed, E1 strengths predictions are sensitive to the choice of $N_{s h}$. More precisely, they are shifted towards lower energies when $N_{s h}$ is increased and also converge with increasing $N_{s h}$. It is well illustrated in Fig. 1(a) by the fact that for $N_{s h}=11$ and 13, QRPA strengths are very close to each other, as compared to $N_{s h}=9$. Remembering the known overestimation of the order of $2 \mathrm{MeV}$ of the energy position of the QRPA E1 strength function in ${ }^{238} \mathrm{U}$ [4], it is important to be able to disentangle between model limitations and lack of convergence. We therefore display in Fig. 1(b), a zoom on the energy peak region for ${ }^{238} \mathrm{U}$ to better estimate the peak position as a function of $N_{s h}$. In this case, we have also considered the highest possible $N_{s h}$ value of 17 , and doing so, confirm the convergence observed in Fig. 1(a). It is also worth to mention that a $60 \mathrm{MeV}$ cutoff is used in Fig. 1(b) to remain within reasonable computation time. As can be observed, the energy shifts of the predicted strengths are much lower than $2 \mathrm{MeV}$ which proves that the $2 \mathrm{MeV}$ shift noticed in Ref. [4] stems from model limitations rather than convergence issues. We have performed such analysis for several nuclei and it has been found that this conclusion was valid for all nuclei and that it was thus possible to obtain rather well converged predictions within computing time compatible with systematic calculation of E1 strength functions [5].

\section{Comparison with experiment}

All nuclei for which experimental photoabsorption data have been analyzed and compiled in the IAEA RIPL-3 library [1] are now considered. In this compilation, most of the nuclei are not even-even and thus cannot be, strictly speaking, compared with our predictions limited here to even-even nuclei. For odd-mass (resp. odd odd) nuclei, we therefore perform theoretical calculations performed for the 2 (respectively 4) neighboring even-even nuclei. Moreover, the analysis has been performed fitting experimental photoabsorption data with one Lorentzian function or two if necessary. We thus use two methods to compare our predictions with experiment. When a single Lorentzian is recommended in RIPL, we compare its peak energy with the centroid of the total strength, obtained by summing the $K^{\pi}=0^{-}$and $K^{\pi}=1^{-}$(where $K$ is the spin projection in axial symmetry) theoretical components
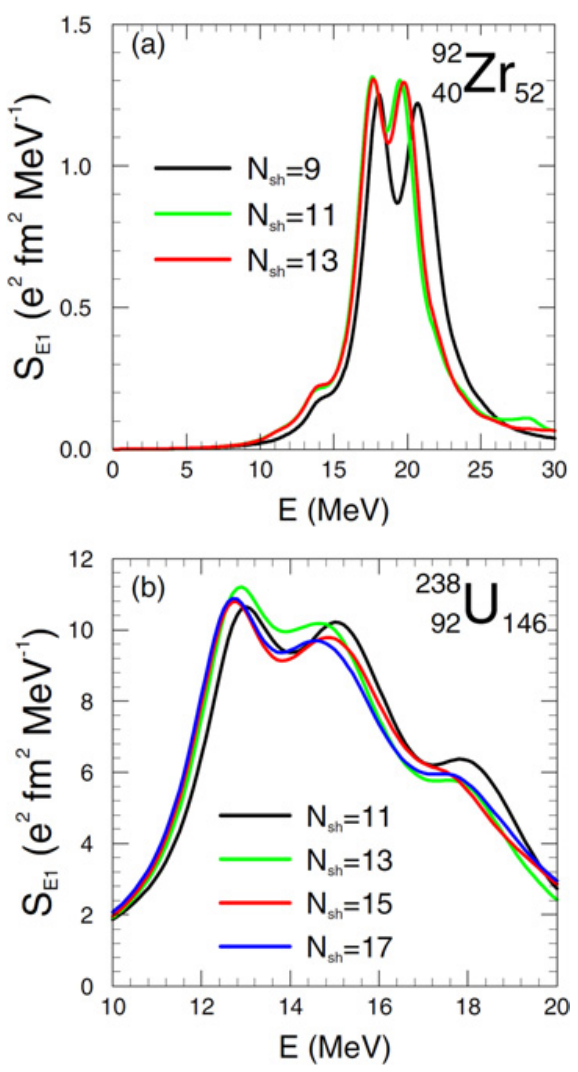

Figure 1. E1 strength as function of the excitation energy for various choices of $N_{s h}$. (a) Configuration without any cutoff. (b) Configuration with a $60 \mathrm{MeV}$ cut-off.

if the nucleus is predicted to be deformed. When two Lorentzian functions are recommended in RIPL, we compare the energy of the lowest (respectively, highest) peak with the centroid of the $K^{\pi}=0^{-}$(respectively, $1^{-}$) component for nuclei theoretically predicted to be prolate and with the centroid of the $K^{\pi}=1^{-}$(respectively, $0^{-}$) component for nuclei theoretically predicted to be oblate, following the opposite ordering of the $0^{-}$and $1^{-}$strengths observed for prolate and oblate nuclei for dipole [4] as well as quadrupole resonances $[8,9]$.

The comparisons are shown in Fig. 2. As can be observed, our predictions display a qualitatively good mass dependence compared to experimental data, a feature particularly striking in the rare earth region. However, the predicted centroid energies are systematically overestimating the fitted values by approximately $2 \mathrm{MeV}$, as already observed for ${ }^{238} \mathrm{U}$ [4]. Indeed, our QRPA calculations do not account for the effects related to qp configurations involving more than 2-qp states or to the phonon coupling which have been shown to shift by a few $\mathrm{MeV}$ the centroid energies to lower energies with respect to the standard QRPA values [10-18].

\section{Strength functions folding}

Our theoretical predictions provide a rather satisfactory description of the GDR centroid of the E1 mode provided a shift of the order of $2 \mathrm{MeV}$ is applied. However, the GDR is known to have a relatively large width and therefore a finite lifetime and for a proper description of experimental data, it is necessary to go beyond the QRPA scheme. Several microscopic theories such as second RPA [19,20], 


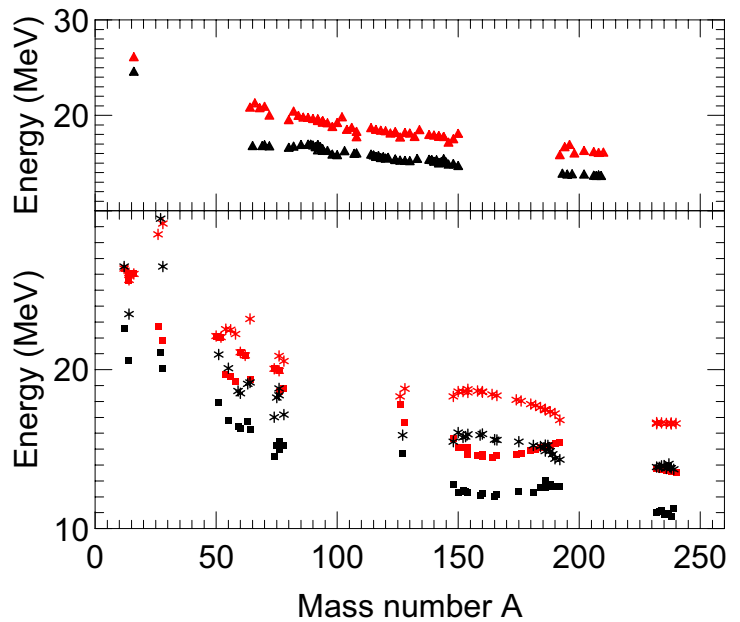

Figure 2. Experimental (black) and theoretical (red) E1 strength centroid energies as function of the mass number. In the upper panel, nuclei for which only one Lorentzian function is used to fit photoabsorption measurements are shown. In the lower panel, two Lorentzian functions have been used and the squares (resp. stars) correspond to the highest (resp. lowest) energy.

particle-vibration coupling [21] or quasiparticle-phonon model [22-24]) explain the location and width of the GDR, but for simplicity and applicability to a large number of nuclei of astrophysical interest, we here restrict ourselves to a semiempirical broadening of the GDR, obtained by folding the QRPA B(E1) distribution by a normalized Lorentzian function,

$$
L(E, \omega)=\frac{1}{\pi} \frac{\Gamma E^{2}}{\left[E^{2}-(\omega-\Delta)^{2}\right]^{2}+\Gamma^{2} E^{2}},
$$

whose width $\Gamma$ and energy shift $\Delta$ can be adjusted on experimental data, such that the E1 $\gamma$-ray strength function $S_{E 1}(E)\left(\right.$ in $\left.e^{2} \mathrm{fm}^{2} \mathrm{MeV}^{-1}\right)$

$$
S_{E 1}(E)=\sum_{n} L\left(E, \omega_{n}\right) B(E 1)\left(\omega_{n}\right),
$$

becomes a continuous function of the energy. Due to the phenomenological character of such an approach, three different prescriptions are adopted to estimate $\Delta$ and $\Gamma$. The simplest model (Model 0) uses $\Delta=2 \mathrm{MeV}$ and $\Gamma=2.5 \mathrm{MeV}$ to globally reproduce experimental photoabsorption data. For the two other models, the width $\Gamma$ is adjusted on each photoabsorption cross section available experimentally with an energy shift taken energydependent following the simple relation $\Delta(\omega)=\Delta_{0}+$ $\Delta_{4 q p}(\omega)$, where $\Delta_{0}$ is a constant shift due to the coupling between $q p$-states and phonons and the quantity $\Delta_{4 q p}(\omega)$ is an extra shift which empirically describes the effect of complex configurations and for this reason is taken to be proportional to the number of 4- $q p$ states which varies with the excitation energy $\omega$ and obviously depends on the nucleus considered. Two different prescriptions are considered to estimate this extra energy shift. The first approximation (Model 1) assumes that $\Delta_{4 q p}^{K}=\delta_{4 q p} \times$ $n_{4 q p}^{K}(\omega) / n_{4 q p}^{K}(\omega=30 \mathrm{MeV})$ where $\delta_{4 q p}$ is a parameter adjusted on experimental photoabsorption cross sections, while the second approximation (Model 2) takes into account the number of 4- $q p$ states relative to the number

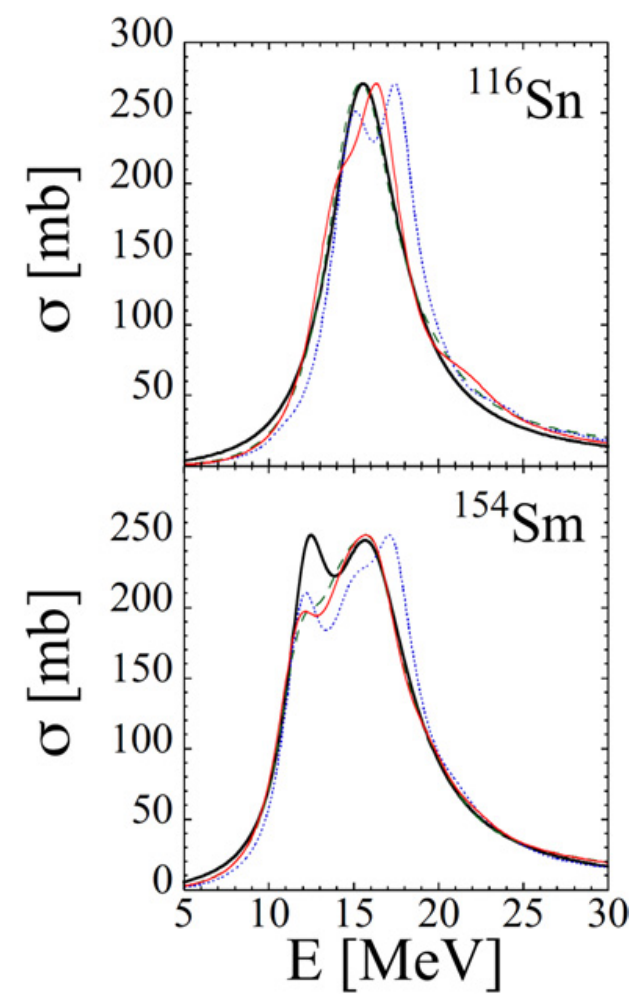

Figure 3. Comparison for a spherical $\left({ }^{116} \mathrm{Sn}\right)$ and a deformed $\left({ }^{154} \mathrm{Sm}\right)$ nucleus between the experimental photoabsorption cross section [1] (black solid line) and the fits corresponding to Model 0 (dotted blue line), Model 1 (green dashed line) and Model 2 (red solid line).

of 2-qp states at the excitation energy $\omega$ and reads $\Delta_{4 q p}^{K}=$ $\delta_{4 q p} \times n_{4 q p}^{K}(\omega) / n_{2 q p}^{K}(\omega)$.

Typical fits obtained with these 3 models are illustrated in Fig. 3. Generally speaking, Model 0 globally reproduces experimental data but, because of its simplicity, provides worse fits than the two other models. With this model, the QRPA strength of some spherical nuclei also displays a second peak which is not observed experimentally and globally disappears when an energy-dependent shift is introduced since it is merged with the low energy one. The fact that each parameter in Model 1 and 2 has been adjusted for each nucleus appears clearly in Fig. 4. One cannot notice any trivial $N$ or $A$ behavior so that, for experimentally unknown nuclei, an interpolation procedure is adopted to estimate the $\Delta_{0}, \Gamma$ and $\delta_{4 q p}$ parameters that cannot be deduced from experimental data. The same holds for odd- $A$ and odd-odd nuclei for which no QRPA calculations has been performed. In this case, due to the relatively smooth variation of GDR between neighboring nuclei [25], we also adopt an interpolation procedure from the neighboring even-even nuclei based on a geometrical mean and a normalization of the strength to the interpolated energy-weighted sum rule. This procedure gives satisfactory results as shown in Fig. 5. As for eveneven nuclei, a second peak is found when using Model 0 which disappears with Models 1 and 2.

The low-energy $\gamma$-ray strength function is known to be crucial for photo-nuclear reaction description. Although our various models fits have been mainly driven by the high energy part of the GDR, around 10 to $15 \mathrm{MeV}$, it is interesting to observe in Fig. 6 


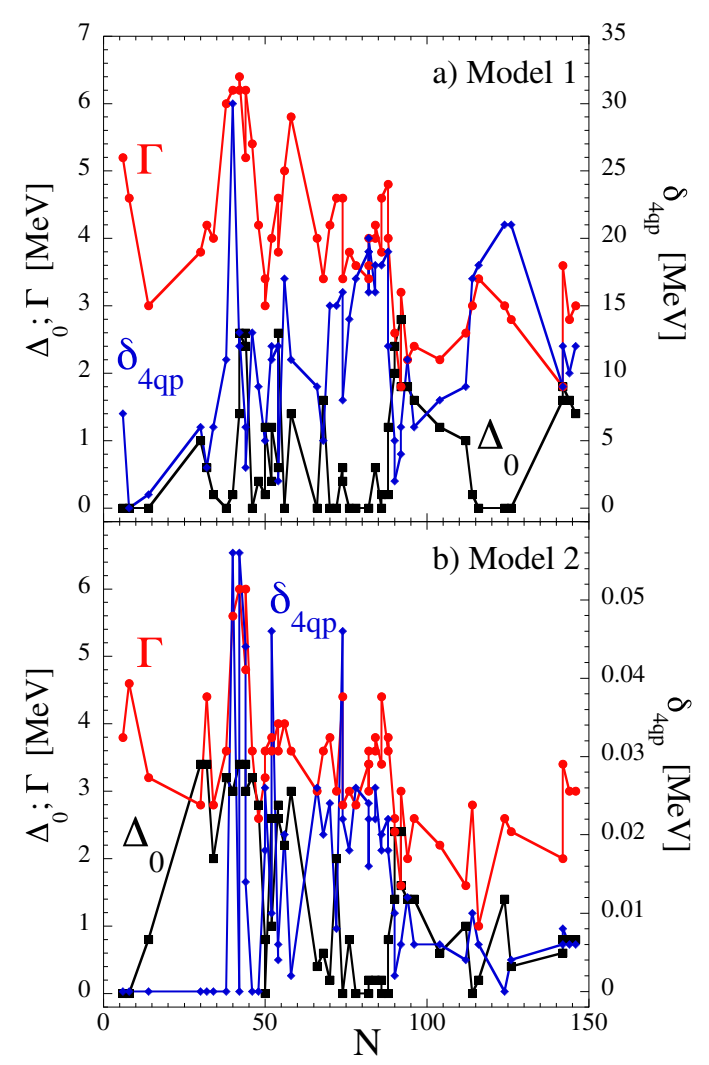

Figure 4. Values of the three parameters $\Delta_{0}$ (squares), $\Gamma$ (circles) and $\delta_{4 q p}$ (diamonds) adjusted on experimental photoabsorption cross sections as a function of the neutron number $\mathrm{N}$.

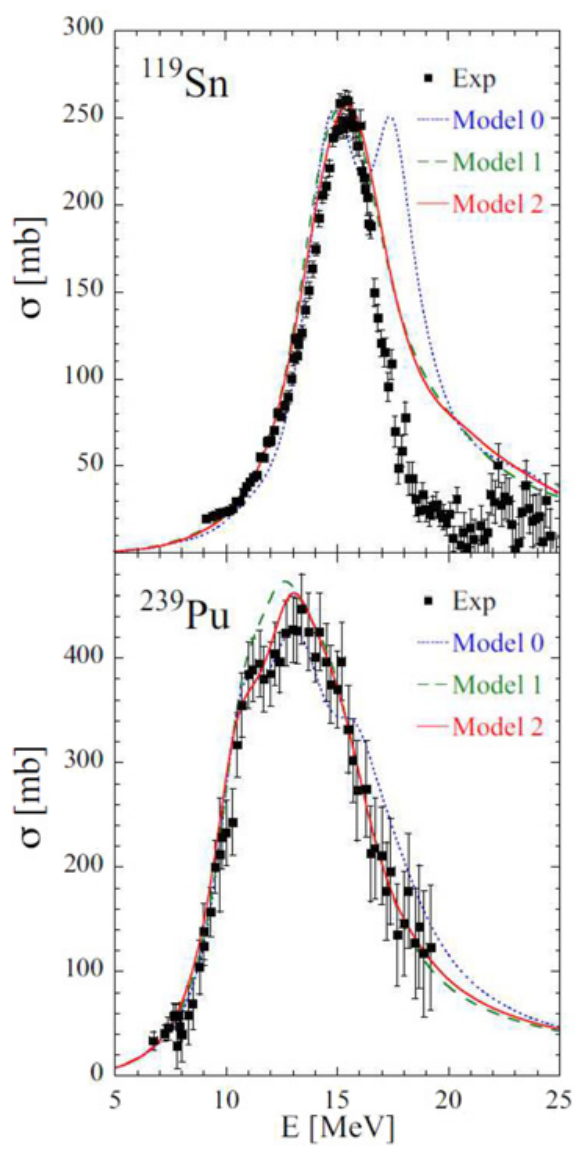

Figure 5. Comparison between experimental photoabsorption cross sections $[26,27]$ (squares) and the QRPA ones for 2 oddA nuclei.

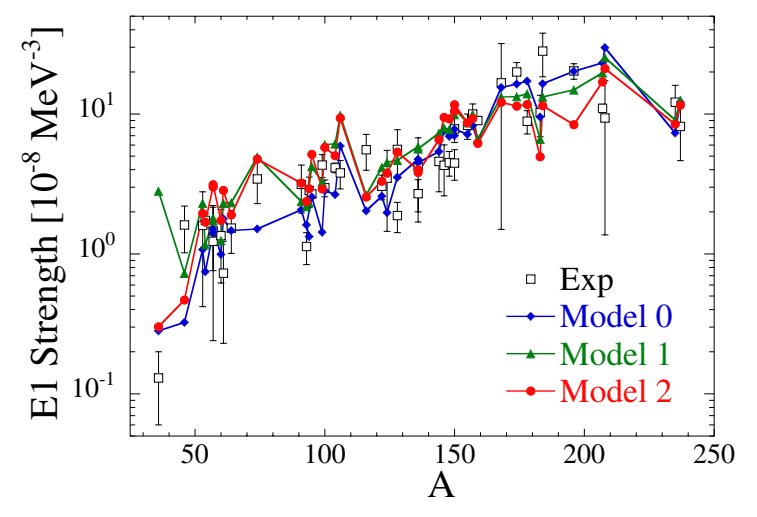

Figure 6. Comparison of the QRPA low-energy E1-strength functions with the experimental compilation [1].

that our predictions also display a rather good agrement with low energy data. In this figure, the compilation of recommended values of experimental E1 strength functions at energies ranging from 4 to $8 \mathrm{MeV}$ [1] for nuclei from ${ }^{36} \mathrm{Cl}$ up to ${ }^{239} \mathrm{U}$, which includes resolvedresonance measurements, thermal-captures measurements and photo-nuclear data, is compared with our three models. They all reproduce rather well the trend of the strengths with respect to the mass number within the error bars and the difference between theory and experiment remains within one order of magnitude except for the two $A<50$ nuclei considered here. The rms deviation on the 44 theoretical to experimental ratios amounts to $1.78,2.02$ and 1.84 for Models 0,1 and 2, respectively. The fact that Model 1 and 2 are not necessarily better than Model 0 reflects the fact that their parameters have been adjusted to fit GDR data rather than the low energy part of the QRPA spectrum.

\section{Exotic neutron-rich nuclei}

We now discuss the predictive power of our approach. Indeed, since we have been using some phenomenological ingredients to reproduce reaction observables, it is worth studying the sensitivity of our predictions far from the valley of stability as function of the model used. For this reason, we compare in Fig. 7 various model predictions for the ${ }^{115-155} \mathrm{Sn}$ isotopes. Note that Model 1 and 2 provide rather similar results and only Model 1 is therefore plotted. The GDR predictions are clearly different but all microscopic (or semi-microscopic) approaches exhibit some extra strength around $5 \mathrm{MeV}$ for $\mathrm{Sn}$ isotopes above the $N=82$ shell closure while the Lorentzian approach cannot predict, by definition, such an additional strength at low energy. This low-lying strength is however quite different in the case of the Skyrme+QRPA [3] results, which predicts pygmy resonances more enhanced than the Gogny ones. For nuclei close to the valley of $\beta$-stability, the microscopic E1 strength functions look rather similar to the phenomenological Lorentzian.

Such differences of course have large impact on cross section predictions. These differences are illustrated plotting in Fig. 8 the Maxwellian-averaged neutron capture rate calculated with the TALYS code [28-31] using the $\gamma$-ray strength obtained with our three models as well as with the Generalized Lorentzian (GLO) model and the 


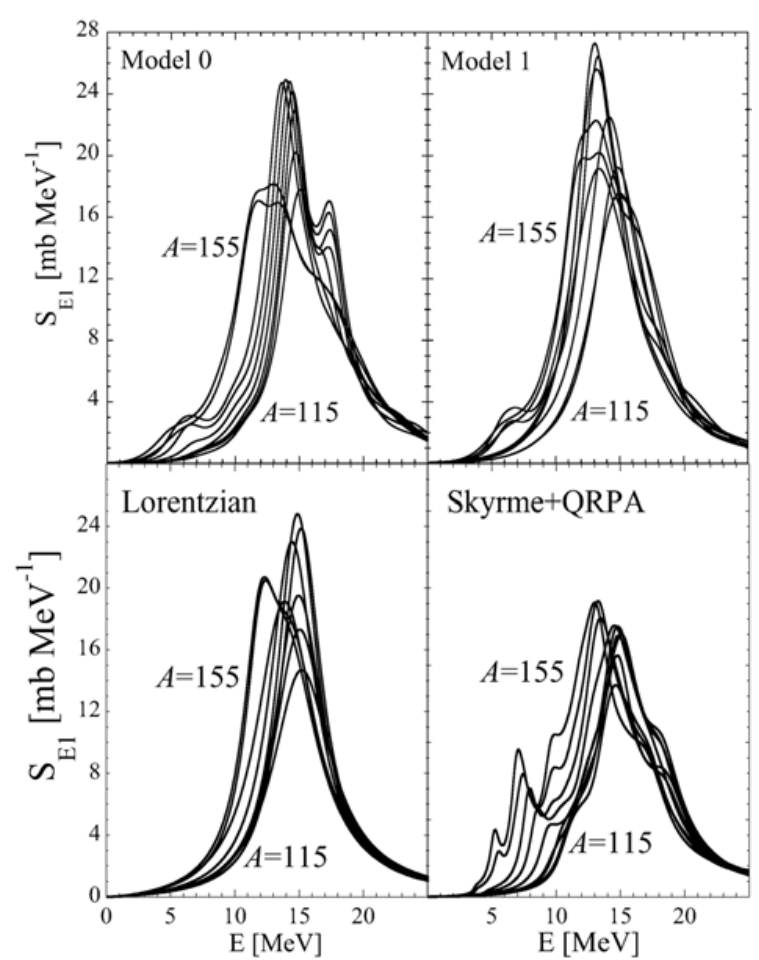

Figure 7. Comparison between various strength function predictions for the ${ }^{115-155} \mathrm{Sn}$ isotopes. Model 0 and 1 correspond to the current work, the Lorentzian model is the Generalized Lorentzian (GLO) of Ref. [1] and the Skyrme+QRPA correspond to that of Ref. [3].

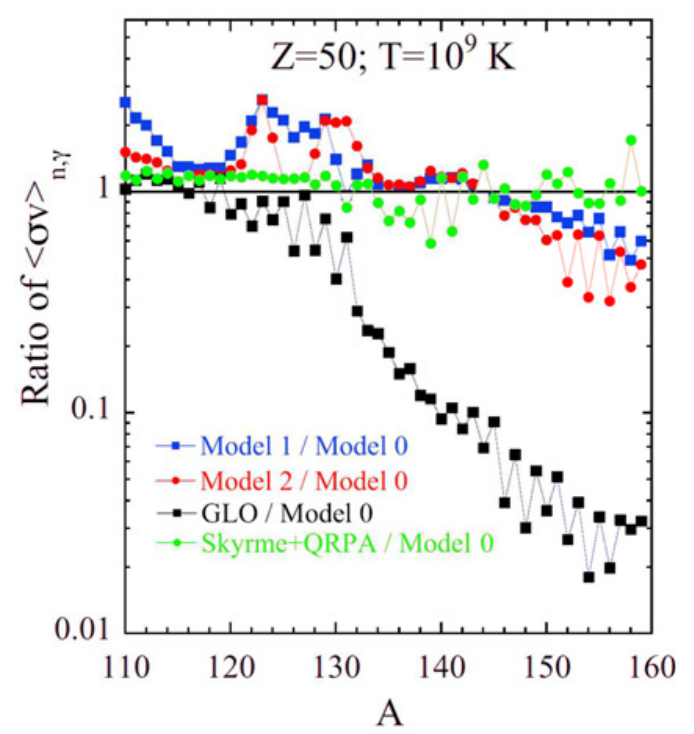

Figure 8. Ratio of the Maxwellian-averaged radiative neutron capture rate (at a temperature of $10^{9} \mathrm{~K}$ ) for the $\mathrm{Sn}$ isotopes obtained with four models with respect to Model 0 .

Skyrme-HFB+QRPA model [3]. For consistency, the D1M masses [7] and the HFB plus combinatorial nuclear level densities based on the D1M single-particle and pairing properties [32] are adopted. As can be observed, the three current models used to fold the original D1M+QRPA strength predict radiative neutron capture rates within a factor of 2, even for the most exotic nuclei. They also agree rather well with those based on the Skyrme-HFB plus QRPA in the whole isotopic chain. On the contrary, for exotic neutron-rich $\mathrm{Sn}$ isotopes our predictions give rise to reaction rates about 30 times larger than those obtained with the GLO model, a feature directly related to the total absence of the pygmy component in the GLO model.

\section{Conclusion}

Large scale calculations of E1 $\gamma$-ray strength functions for even-even nuclei have been conducted within the axiallysymmetric-deformed HFB+QRPA approach in a fully consistent way using the finite-range Gogny interaction. The convergence of the numerical calculation has been analyzed with respect to the size of the basis (number of harmonic oscillator shells) and the 2-qp excitation energy. Practical choices have thus been made for large scale calculations optimizing both the minimization of the computational cost and the convergence. A systematic energy shift of the predicted E1 strength compared to experimental data of the order of $2 \mathrm{MeV}$ has been observed and several phenomenological options have been considered to correct this discrepancy in the folding procedure of the $\mathrm{B}(\mathrm{E} 1)$ discrete distributions, needed to produce gamma-ray strength functions used as input for reaction models. All prescriptions give satisfactory description of experimental data and provide globally similar E1 strength functions. When used to predict astrophysical capture rates, the results with all prescriptions do not differ by more than a factor of 2 , even far away from the valley of $\beta$-stability, while more than on order of magnitude difference is observed in comparison with more traditional analytical approaches usually adopted in nuclear reaction codes. The present approach can be improved in several directions. First, the interpolation employed to obtain the E1 strength for odd nuclei should be replaced by a fully microscopic QRPA calculation of odd system. Second, the folding procedure should be improved by developing a microscopic particlephonon coupling calculation. Third, dynamic deformation should be considered when it is different from the HFB one. The encouraging results obtained for E1 strengths have already been extended to magnetic dipole M1 strengths [33].

In sum, in parallel to the nuclear masses [7] and the nuclear level densities [32], we are following a project which consists in including, one by one, the ingredients required to perform microscopic cross sections calculations on the basis of an optimized Gogny interaction. Working along such a path is a way to obtain, in the future, satisfactory cross section evaluations and predictions on the basis of reliable and accurate microscopic inputs.

S.G. is F.N.R.S. research associate. We acknowledge PRACE for awarding us access to resource CURIE based in FRANCE at TGCC-CEA.

\section{References}

[1] R. Capote, et al., Nuclear Data Sheets 110, 3107 (2009). See also http://www-nds.iaea.org/ RIPL-3

[2] S. Goriely and E. Khan, Nucl. Phys. A 706, 217 (2002) 
[3] S. Goriely, E. Khan, and M. Samyn, Nucl. Phys. A 739, 331 (2004)

[4] S. Péru et al., Phys. Rev. C 83, 014314 (2011)

[5] M. Martini et al., Phys. Rev. C 94, 014304 (2016)

[6] J.F. Berger, M. Girod, D. Gogny, Comp. Phys. Comm. 63, 365 (1991)

[7] S. Goriely et al., Phys. Rev. Lett. 102, 242501 (2009)

[8] S. Péru and H. Goutte, Phys. Rev. C 77, 044313 (2008)

[9] G. Scamps and D. Lacroix, Phys. Rev. C 89, 034314 (2014)

[10] P. Papakonstantinou and R. Roth, Phys. Lett. B 671, 356 (2009)

[11] D. Gambacurta et al., Phys. Rev. C 84, 034301 (2011)

[12] D. Gambacurta et al., Phys. Rev. C 86, 021304 (2012)

[13] D. Gambacurta et al., Phys. Rev. C 93, 024309 (2016)

[14] G. Colo and P.F. Bortignon, Nucl. Phys. A 696, 427 (2001)

[15] S. Kamerdzhiev et al., Phys. Rep. 393, 1 (2004)

[16] D. Sarchi et al., Phys. Lett. B 601, 27 (2004)

[17] A. Avdeenkov et al., Phys. Rev. C 83, 064316 (2011)
[18] O. Achakovskiy et al., Phys. Rev. C 91, 034620 (2015)

[19] C. Yannouleas et al. Nucl. Phys. A 397, 239 (1983)

[20] C. Yannouleas, Phys. Rev. C 35, 1159 (1987)

[21] G.F. Bertsch et al., Rev. Mod. Phys. 55, 287 (1983)

[22] V.G. Soloviev, Theory of complex nuclei (Oxford: Pergamon Press, 1976)

[23] V.G. Soloviev et al., Nucl. Phys. A 304, 503 (1978)

[24] N. Tsoneva and H. Lenske, Phys. Rev. C 77, 024321 (2008)

[25] Photonuclear data for applications; cross sections and spectra, IAEA-Tecdoc-1178 (2000)

[26] S.C. Fultz et al., Phys. Rev. 186, 1255 (1969)

[27] M.A.P.V. De Moraes, M.F. Cesar, Physica Scripta 47, 519 (1993)

[28] S. Hilaire, A.J. Koning and S. Goriely, Journal of the Korean Physical Society 59, 767 (2011)

[29] S. Goriely, S. Hilaire and A.J. Koning Astronomy and Astrophysics 487, 767 (2008)

[30] A.J. Koning, S. Hilaire and M.C. Duijvestijn Nucl. Data for Science and technology (EDP Sciences, eds Bersillon et al.) 211 (2008)

[31] A.J. Koning and D. Rochman, Nuclear Data Sheets 113, 2841 (2012)

[32] S. Hilaire, M. Girod, S. Goriely S and A.J. Koning, Phys. Rev. C 86, 064317 (2012)

[33] S. Goriely et al., Phys. Rev. C 94, 034306 (2016) 